\title{
Laminar Flow Induced by a Point Source of Heat
}

\author{
Howard R. Baum \\ Instifute for Applied Technology, National Bureau of Standards, Washington, D.C. 20234
}

(April 18, 1977)

\begin{abstract}
An asymptotic description of the laminar flow pattern generated by a point source of heat is derived. Existing solutions of the boundary layer equations are shown to be valid in a limited domain where buoyancy forces are dominant. These solutions are supplemented by new results for the buoyant plume and are matched asymptotically to solutions valid everywhere outside the plume. Composite analytical formulae for the pressure, enthalpy and velocity fields are obtained and applied to the computation of the streanline pattern, pressure, and enthalpy distribution for Prandtl numbers 0.7 and 1.0.
\end{abstract}

Key words: Analysis; bouyancy; laminar flow; plume; pressure distribution; streamlines; temperature distribution.

\section{Introduction}

The flow induced by an isolated heat source is a prototype for many buoyancy driven flows. The motion is initiated because the heated gas adjacent to the source becomes less dense and rises. The rising hot gas column draws in cool stationary fluid from the surroundings, reducing the excess temperature and hence the relative buoyancy in the moving fluid. The resulting steady flow pattern is a long narrow vertical plume of heated fluid rising through a nearly quiescent medium. The intuitive picture sketched above has led most previous investigators to study the problem by applying the boundary layer approximation to the equations of motion appropriate to a weakly buoyant flow (the Boussinesq equations). The procedure results in a system of ordinary differential equations which has been solved many times. Thus, Schuh [1] ${ }^{1}$ obtained numerical results for a fluid of Prandtl number $(\operatorname{Pr})$ 0.7. Yih [2] gave closed form solutions for $\operatorname{Pr}=1$ and $P r=2$. Fujii [3] presented numerical results for $\operatorname{Pr}=0.01,0.7$, and 10, in addition to the closed form solutions for $P r=1$ and 2. Most o Fujii's results were repeated independently by Brand and Lahey [4]. A useful survey of natural convection phenomena containing these and related references is given by Gebhart [5]

The above analyses cannot describe the entire flow pattern, however. The fluid entrained in the plume must come from somewhere; hence the surrounding fluid cannot be at rest. Moreover, the pressure cannot be constant, not even within the plume. In fact, inspection of the solutions to the boundary layer equations raises a question as to the sense in which boundary layer theory is applicable to the present problem. The difficulty arises from the fact that the solutions decay algebraically to their ambient values outside the plume. This behavior is very different from the exponential decay which obtains in most boundary layer problems, including the two dimensional (line source) thermal plume [3].

The situation is analogous to that which occurs in the study of the flow induced by a momentum source. The plane momentum jet (line source) was studied using the boundary layer equations by Schlichting [6] and Bickley [7]. An English language account of Schlichting's work may be found in Schlichting [8]. The velocity profile in the plane jet decays exponentially as the ambient fluid is approached. The corresponding boundary layer solution for a point momentum source [6] is again algebraic, as in the problem under consideration. Fortunately, the exact solution of the Navier Stokes equations for a point source of momentum has been given by Landau [9]. The boundary layer solution describes the velocities (but not the pressure distribution) near the axis of symmetry provided the cylindrical coordinates that appear in the solution are reinterpreted as small angle approximations to spherical polar coordinates. However, the flow away from the axis is nontrivial and cannot be obtained from the boundary layer equations.

\footnotetext{
1 Figures in brackets indicate the literature references at the end of this paper.
} 
In the present paper an asymptotic solution for the flow induced by the point heat source is derived. The solution is valid sufficiently far from the source in all directions, unlike the solutions based on the boundary layer equations. The key elements in the solution are the determination of the pressure distribution in the plume and the use of parabolic coordinates. The introduction of parabolic coordinates is suggested by the work of Landau [9] and Goldstein [10] on the point momentum source and flat plate boundary layer, respectively. It permits a simple derivation of the boundary layer form of the equations which apply in the plume region above the heat source. These equations are solved analytically for any Pr of order unity. They lead to an expression for the pressure in the plume, a quantity not previously calculated. The form of the pressure distribution, together with corresponding expressions for the velocity and enthalpy in the plume, allow simple analytic expressions to be obtained for the velocity and pressure distributions in the fluid both inside and outside the plume. Finally, the enthalpy distribution outside the plume is determined and combined with the solution in the plume to produce a result valid in all directions far from the source.

Fendell [11] treated the flow induced by a small heated sphere, and a portion of that analysis is closely related to the work presented here. Fendell derived the boundary layer equations for the velocity and enthalpy in parabolic coordinates, but assumed the pressure to be constant. The flow pattern outside the plurne was determined using an Oseen linearization about the maximum plume velocity. This idea, while attractive in principle, leads to solutions which are qualitatively different from those obtained below. The use of the Oseen linearization in free convection problems appears to be more difficult than is the case in forced convection. Even when forced convection occurs, bouyancy can still introduce difficulties as Wesseling [12] has shown.

\section{The Equations of Motion}

The Boussinesq form of the Navier Stokes equations for the steady flow of a constant property fluid in a gravitational field may be written in vctor invariant form as:

$$
\begin{gathered}
\nabla \cdot \mathbf{u}=0 \\
\nabla\left(\frac{1}{2} u^{2}\right)-\mathbf{u} \mathbf{x} \boldsymbol{\omega}+\frac{1}{\rho} \nabla\left(p-p_{\infty}\right)+\frac{\left(h-h_{\infty}\right)}{h_{\infty}} \mathbf{g}=-\nu \nabla \mathbf{x} \boldsymbol{\omega} \\
\mathbf{u} \cdot \nabla\left(h-h_{\infty}\right)=\frac{\nu}{\operatorname{Pr}} \nabla^{2}\left(h-h_{\infty}\right) \\
\nabla \mathbf{x u}=\boldsymbol{\omega} .
\end{gathered}
$$

The first three of equations (1) are the conservation of mass, momentum, and energy, respectively, while the final equation is a definition of the vorticity $\omega$. The dependent variables are the fluid velocity $\mathbf{u}$, the pressure $p$, and the enthalpy per unit mass $h$. The fluid properties are the density $\rho$, the kinematic viscosity $\nu$, and the Prandtl number $\operatorname{Pr}$. The quantity $\mathbf{g}$ is the gravitational acceleration, and the subscript $\infty$ denotes the value in the undisturbed fluid far from the source. The boundary conditions are:

$$
\begin{gathered}
\mathbf{u}=p-p_{\infty}=h-h_{\infty}=0 ; \mathbf{r} \rightarrow \infty \\
\int\left\{\rho \mathbf{u} \cdot \mathbf{n}\left(h-h_{\infty}\right)-\frac{\nu}{P r} \nabla h \cdot \mathbf{n}\right\} d s=2 \pi Q .
\end{gathered}
$$

The boundary conditions (2) assume that the heat source of strength $2 \pi Q$ is located at the origin of coordinates $\mathbf{r}=0$. The integral is taken over an arbitrary surface which encloses the origin.

The motion is symmetric about an axis parallel to the gravity vector $\mathrm{g}$ which passes thru the source. Let $r$ denote the radial distance from the axis of symmetry and $z$ be the distance above the source measured along the axis. The buoyancy dominated region lies above the source with a radial extent given by [3]:

$$
r \sim(z)^{1 / 2} .
$$


The motion induced everywhere else is essentially irrotational. The configuration is illustrated schematically in figure 1. As mentioned earlier, it is convenient to introduce parabolic coordinates defined as follows:

$$
\begin{aligned}
& \xi_{1}=\left\{\sqrt{r^{2}+z^{2}}+z\right\}^{1 / 2} \\
& \xi_{2}=\left\{\sqrt{r^{2}+z^{2}}-z\right\}^{1 / 2} .
\end{aligned}
$$

Let $\mathbf{i}_{1}, \mathbf{i}_{2}$, be unit vectors in the direction of $\xi_{1}, \xi_{2}$; and $\mathbf{i}_{3}$ be defined by:

$$
\mathbf{i}_{3}=\mathbf{i}_{1} \mathbf{x} \mathbf{i}_{2} \text {. }
$$

The first of equations (1) can be used to define a stream function $\Psi$.

$$
\begin{aligned}
& \mathbf{u}=u \mathbf{i}_{1}+v \mathbf{i}_{2} \\
& u=\frac{1}{\sqrt{\xi_{1}^{2}+\xi_{2}^{2}}} \frac{1}{\xi_{1} \xi_{2}} \frac{\partial \Psi}{\partial \xi_{2}} \\
& v=\frac{-1}{\sqrt{\xi_{1}^{2}+\xi_{2}^{2}}} \frac{1}{\xi_{1} \xi_{2}} \frac{\partial \Psi}{\partial \xi_{1}} .
\end{aligned}
$$

The conservation of momentum (the second of equations (1)) becomes

$$
\begin{aligned}
& \mathbf{i}_{1}\left\{u \frac{\partial u}{\partial \xi_{1}}+v \frac{\partial v}{\partial \xi_{1}}-\sqrt{\xi_{1}{ }^{2}+\xi_{2}{ }^{2}} v \omega+\frac{1}{\rho} \frac{\partial}{\partial \xi_{1}}\left(p-p_{\infty}\right)\right\} \\
& +\mathbf{i}_{2}\left\{u \frac{\partial u}{\partial \xi_{2}}+v \frac{\partial v}{\partial \xi_{2}}+\sqrt{\xi_{1}{ }^{2}+\xi_{2}{ }^{2}} u \omega+\frac{1}{\rho} \frac{\partial}{\partial \xi_{2}}\left(p-p_{\infty}\right)\right\} \\
& =\mathbf{i}_{1}\left\{\xi_{1} g\left(\frac{h}{h_{\infty}}-1\right)-\frac{\nu}{\xi_{1} \xi_{2}} \frac{\partial}{\partial \xi_{2}}\left(\xi_{1} \xi_{2} \omega\right)\right\} \\
& -\mathbf{i}_{2}\left\{\xi_{2} g\left(\frac{h}{h_{\infty}}-1\right)-\frac{\nu}{\xi_{1} \xi_{2}} \frac{\partial}{\partial \xi_{1}}\left(\xi_{1} \xi_{2} \omega\right)\right\} .
\end{aligned}
$$

The terms on the left-hand side of eq (5) are the inertial and pressure contributions to the momentum balance while the right-hand side contains the effect of buoyancy and viscosity. The quantity $g$ is the magnitude of $\mathbf{g}$, and $\omega$ is related to the vorticity $\boldsymbol{\omega}$ and the stream function $\Psi$ as follows:

$$
\begin{aligned}
\boldsymbol{\omega} & \equiv \mathbf{i}_{3} \boldsymbol{\omega} \\
& =\frac{-\mathbf{i}_{3}}{\left(\xi_{1}{ }^{2}+\xi_{2}{ }^{2}\right)}\left\{\frac{\partial}{\partial \xi_{1}}\left(\frac{1}{\xi_{1} \xi_{2}} \frac{\partial \Psi}{\partial \xi_{1}}\right)+\frac{\partial}{\partial \xi_{2}}\left(\frac{1}{\xi_{1} \xi_{2}} \frac{\partial \Psi}{\partial \xi_{2}}\right)\right\} .
\end{aligned}
$$

Finally, the energy equation becomes:

$$
\begin{gathered}
\frac{\partial \Psi}{\partial \xi_{2}} \frac{\partial \Theta}{\partial \xi_{1}}-\frac{\partial \Psi}{\partial \xi_{1}} \frac{\partial \Theta}{\partial \xi_{2}}=\frac{\nu}{\operatorname{Pr}}\left\{\frac{\partial}{\partial \xi_{1}}\left(\xi_{1} \xi_{2} \frac{\partial \Theta}{\partial \xi_{1}}\right)+\frac{\partial}{\partial \xi_{2}}\left(\xi_{1} \xi_{2} \frac{\partial \Theta}{\partial \xi_{2}}\right)\right\} \\
\Theta=h-h_{\infty} .
\end{gathered}
$$

The left-hand side of eq (7) represents the convection of energy due to the fluid motion, while the right-hand side accounts for the diffusion of heat. 


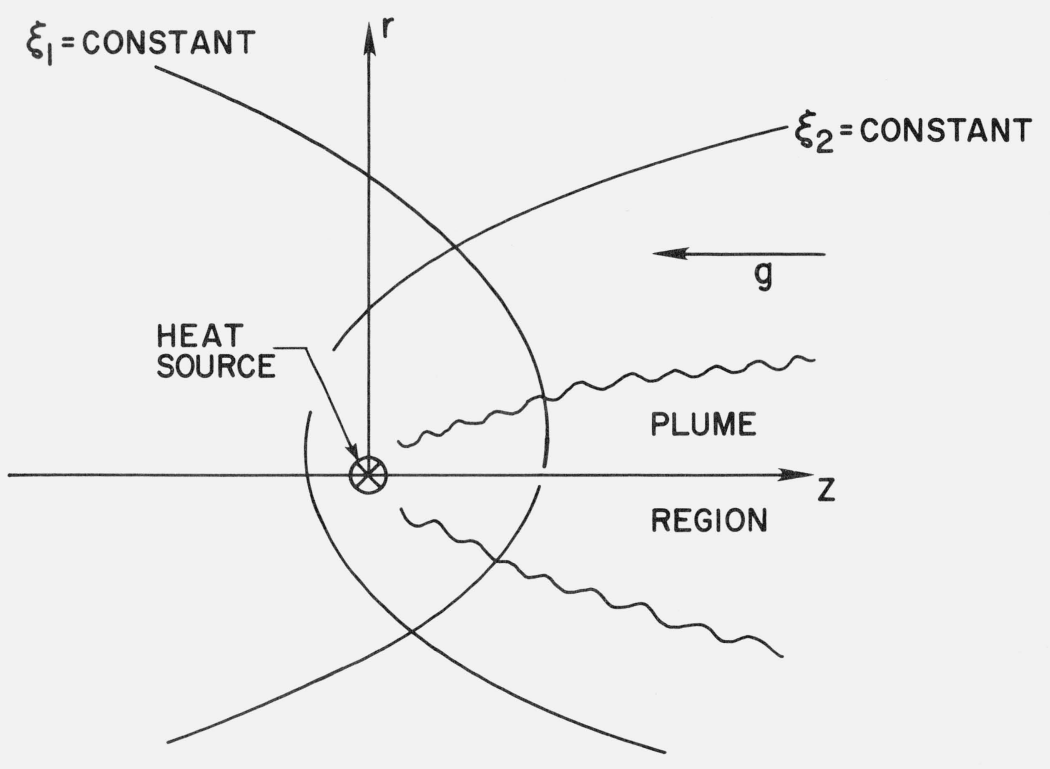

FIgURE 1. Schematic drawing of coordinate geometry and plume orientation.

The solution procedure is based on the fact that the buoyant plume drives the flow. Previous analyses cited above show that the buoyancy dominated region lies in the domain defined by $\xi_{1} \gg \xi_{2}$ (see fig. 1). The appropriate dimensionless variables are:

$$
\begin{aligned}
\eta & =\left(\frac{4 g Q}{\rho h_{\infty} \nu^{3}}\right)^{1 / 4} \xi_{2} \\
\xi & =\left(\frac{g Q}{4 \rho h_{\infty} \nu^{3}}\right)^{1 / 4} \xi_{1} \\
\Psi & =\nu\left(\frac{\rho h_{\infty} \nu^{3}}{g Q}\right)^{1 / 2} \xi^{2} F(\eta) \\
\Theta \equiv h-h_{\infty} & =\frac{Q}{\rho \nu}\left(\frac{g Q}{\rho \nu^{3} h_{\infty}}\right)^{1 / 2} \frac{G(\eta)}{\xi^{2}} \\
p-p_{\infty} & =\frac{g Q}{\nu h_{\infty}} \frac{P(\eta)}{\xi^{2}} .
\end{aligned}
$$

The differing factors of $(4)^{1 / 4}$ appearing in the definitions of $\xi$ and $\eta$ are chosen so the boundary layer equations valid for $\xi \gg \eta$ are the same as those obtained by Fujii [3]. The velocity and vorticity components $u, v$ and $\omega$ are obtained from equations (8) as:

$$
\begin{aligned}
& u=\left(\frac{g Q}{\rho \nu^{3} h_{\infty}}\right)^{1 / 2} \frac{\nu}{\sqrt{1+\frac{1}{4} \frac{\eta^{2}}{\xi^{2}}} \frac{1}{\eta}} \frac{d F}{d \eta} \\
& v=-\left(\frac{g Q}{\rho \nu^{3} h_{\infty}}\right)^{1 / 2} \frac{\nu}{\xi \sqrt{1+\frac{1}{4}} \frac{\eta^{2}}{\xi^{2}}} \frac{1}{\eta} F(\eta) \\
& \omega=\left(\frac{g Q}{\rho \nu^{3} h_{\infty}}\right) \frac{\nu}{\xi\left(1+\frac{1}{4} \frac{\eta^{2}}{\xi^{2}}\right)} \frac{d}{d \eta}\left(\frac{1}{\eta} \frac{d F}{d \eta}\right) .
\end{aligned}
$$


Substitution of equations (8) and (9) into eqs (5) and (7) yields, for $\xi \gg \eta$ :

$$
\begin{gathered}
F \frac{d}{d \eta}\left(\frac{1}{\eta} \frac{d F}{d \eta}\right)+\frac{d}{d \eta}\left[\eta \frac{d}{d \eta}\left(\frac{1}{\eta} \frac{d F}{d \eta}\right)\right]+\eta G=O\left(\frac{1}{\xi}\right) \\
\frac{d}{d \eta}\left\{F G+\frac{\eta}{P r} \frac{d G}{d \eta}\right\}=O\left(\frac{1}{\xi}\right) \\
\frac{d P}{d \eta}+\frac{\eta G}{2}+\frac{F}{\eta} \frac{d}{d \eta}\left(\frac{F}{\eta}\right)=O\left(\frac{1}{\xi^{2}}\right) .
\end{gathered}
$$

Thus, letting $\xi$ approach infinity with $\eta$ held fixed, the right-hand sides of equations (10) may be ignored. The first two of these equations, the $\mathbf{i}_{1}$ component of the momentum equation and the energy equation, have been obtained many times before, but with $\xi$ and $\eta$ replaced by their limiting values for $z \gg r$ :

$$
\begin{gathered}
\xi \sim \sqrt{2 z} \\
\eta \sim \frac{r}{\sqrt{2 z}} .
\end{gathered}
$$

The last of equations (10), which represents the $\mathbf{i}_{2}$ component of the momentum balance, has apparently never been derived previously. Its importance will become apparent below.

The boundary conditions (2) become, to the present degree of approximation:

$$
\begin{gathered}
F=\frac{d F}{d \eta}=0 ; \quad \eta=0 \\
P=\frac{d F}{d \eta}=G=0 ; \quad \eta \rightarrow \infty \\
\int_{0}^{\infty} G \frac{d F}{d \eta} p^{u=1}
\end{gathered}
$$

The conditions at $\eta=0$ insure regularity of the solutions on the axis of symmetry. The conditions at infinity are strictly valid only if the solutions so obtained can be continued analytically, so that equations (2) can be satisfied at infinity for all values of $\xi / \eta$ outside the plume. The integral constraint on the solution is consistent with the other boundary conditions. Physically, it states that the heat generated at the source is primarily convected up the buoyant plume. The role of thermal conduction of heat only enters the overall energy blance (the last of equations (2)) in terms of higher order in $(\xi)^{-1}$.

\section{Solution of the Boundary Layer Equations}

As noted in the introduction, there are many numerical and analytical solutions available to the first two of equations (10) for particular values of the Prandtl number. Rather than obtain additional numerical solutions, approximate analytical solutions are constructed. They are valid for any Prandtl number of order unity, which includes most common gases. The second of equations (10) may be integrated once. Applying the boundary conditions as $\eta$ approaches infinity and defining a new independent variable $y$, the first two of eqs (10) become:

$$
\begin{gathered}
F \frac{d^{2} F}{d y^{2}}+\frac{d}{d y}\left[2 y \frac{d^{2} F}{d y^{2}}\right]+G=0 \\
F G+\frac{2 y}{\operatorname{Pr}} \frac{d G}{d y}=0 \\
\eta^{2}=2 y
\end{gathered}
$$


The method of solution rests on the assumption of the following functional form for $F$ :

$$
F=\frac{\alpha y}{1+\beta y} .
$$

Equation (13) is suggested by the fact that the exact solutions for $\operatorname{Pr}=1$ and $\operatorname{Pr}=2$ have this form. Moreover, the boundary conditions at $y=0$ and the asymptotic behavior at infinity are satisfied for any value of $\operatorname{Pr}$. Finally, eq (13) satisfies the requirement that the solutions be analytic in $y$ near $y=0$, a requirement imposed by the boundary conditions. Once equation (13) is adopted, $G$ can be determined immediately from the second of eqs (12).

$$
\begin{aligned}
G & =g_{0}(1+\beta y)^{-\lambda} \\
\lambda & =\frac{\operatorname{Pr} \alpha}{2 \beta}=\frac{\operatorname{Pr} F(\infty)}{2} .
\end{aligned}
$$

The single additional parameter $g_{o}$ is related to $\alpha$ and $\beta$ by the overall energy balance. Using eqs (13) and (14) in the integral in equation (11):

$$
g_{0}\left(1+\frac{\operatorname{Pr} \alpha}{2 \beta}\right)^{-1}=\beta
$$

The remaining parameters $\alpha$ and $\beta$ are determined by requiring the first of equations (12) (the momentum equation) to be satisfied on the average and at $y=0$. This is a widely used procedure for obtaining approximate solutions in boundary layer theory [8]. At $y=0$ :

Thus:

$$
G(0)+2 \frac{d^{2} F}{d y^{2}}(0)=0
$$

$$
4 \alpha \beta=\dot{g_{0}}
$$

The integrated $\mathbf{i}_{1}$ momentum equation may be put in the form:

$$
\int_{0}^{\infty}\left[G-\left(\frac{d F}{d y}\right)^{2}\right] d y=0
$$

Substituting equations (13) and (14) into this expression:

$$
g_{o}=\frac{\alpha^{2}}{3}\left[\frac{P r \alpha}{2 \beta}-1\right]
$$

Equations (13)-(16) may be readily solved to obtain:

$$
\begin{aligned}
\alpha & =\frac{1}{2}\left\{\frac{3+\sqrt{1+24 \operatorname{Pr}}}{2}\right\}^{1 / 2} \\
\beta & =\frac{\operatorname{Pr}}{2}\left\{\frac{3+\sqrt{1+24 \operatorname{Pr}}}{2}\right\}^{1 / 2}[1+\sqrt{1+24 P r}]^{-1} \\
g_{0} & =\frac{\operatorname{Pr}}{2}[3+\sqrt{1+24 P r}][1+\sqrt{1+24 P r}]^{-1} \\
\lambda & =\frac{1}{2}[1+\sqrt{1+24 P r}]
\end{aligned}
$$

Equations (13), (14) and (17) constitute the approximate solution for $F$ and $G$. The solution is exact for $\operatorname{Pr}=1$ and 2 . Its accuracy was tested by comparing the present results with those of Fujii [3] for $\operatorname{Pr}=0.7$. The comparison is shown in the following table. 


\begin{tabular}{ccc}
\hline \hline Quantity & Fujii [3] & Present \\
\hline$\frac{d F}{d y}(0)$ & 0.938 & 0.950 \\
$F(\infty)$ & 7.528 & 7.456 \\
$G(0)$ & .481 & .484 \\
\hline
\end{tabular}

The entire velocity profile was also plotted against that given by Fujii, but given the smallscale of the figures in [3] it was impossible to display the difference. Finally, it should be noted that Fujii estimated his numerical computations to be accurate to about one percent. Thus, the present solution is about as accurate as that of Fujii for Prandtl numbers of order unity. The Prandtl number restriction is necessary because the momentum and thermal plumes must be of comparable thickness for the above scheme to work. When $\operatorname{Pr} \gg 1$, the momentum plume is much thicker than the thermal plume; while the reverse is true when $\operatorname{Pr} \ll 1$.

The pressure variation within the plume may now be determined from the last of eqs (10). Using eqs (11), (13) and (14), the following expression is obtained:

$$
P=\frac{g_{o}}{2 \beta(\lambda-1)}(1+\beta y)^{-(\lambda-1)}-\frac{1}{4 y}\left(\frac{\alpha y}{1+\beta y}\right)^{2} .
$$

The function $P(\eta)$ is plotted in figure 2 for $\operatorname{Pr}=0.7$ and 1.0. The profiles shown approximate the radial dependence of the pressure near the axis of symmetry far from the source. The

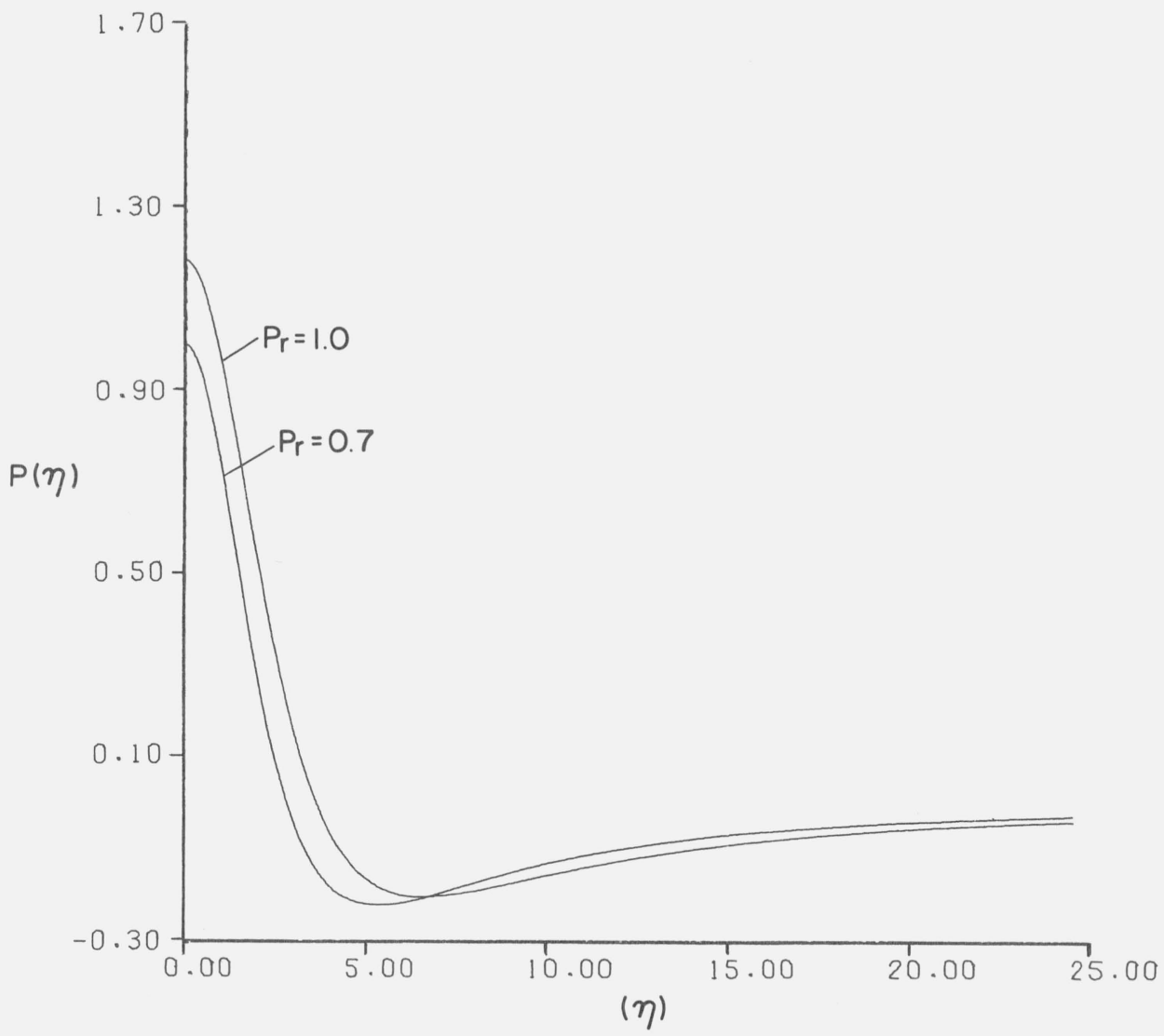

FIGURE 2. The function $\mathrm{P}(\eta)$ describing the pressure profile inside the plume region. 
initial drop in pressure as $\eta$ decreases from infinity is due to the inertial acceleration of the fluid as it enters the plume. The motion here is effectively irrotational, as will be shown in the following section. The pressure rise near the center is due to the buoyancy contribution, the first term in eq (18). A pressure rise near the axis of symmetry is also necessary on geometrical grounds as a means of turning the fluid from an inclined to a nearly vertical direction. A more complete discussion of the flow pattern and pressure distribution must await the calculation of these quantities in the region outside the plume where the inequality $\xi \gg \eta$ does not hold.

\section{The Uniformly Valid Pressure and Velocity Fields}

The determination of the pressure and velocity fields outside the plume can be separated from the calculation of the enthalpy. This separation is possible because the motion in this region is irrotrational. This may be verified by noting that the boundary layer solutions take the following form as $\eta \rightarrow \infty$.

$$
\begin{aligned}
& F \sim \frac{\alpha}{\beta}=F(\infty) \\
& G \sim g_{o}\left(\frac{\beta}{2} \eta^{2}\right)^{-\lambda} \equiv G(\infty)(\eta)^{-P_{\tau F}(\infty)} \\
& P=-\frac{1}{2}\left(\frac{F(\infty)}{\eta}\right)^{2} .
\end{aligned}
$$

The last of eqs (19) follows from the fact that $\lambda>2$ for the $\operatorname{Pr}$ range of interest. Substituting eqs (19) into eqs (8) and (9) the stream function and vorticity become:

$$
\begin{aligned}
& \Psi=\nu\left(\frac{\rho h_{\infty} \nu^{3}}{g Q}\right)^{1 / 2} \xi^{2} F(\infty) \\
& \omega=0 .
\end{aligned}
$$

Thus, the asymptotic form of the solution valid in the plume is an irrotational flow. Now any irrotational flow is an exact solution of the Navier Stokes equations (1) provided the buoyancy terms may be ignored. Under these circumstances, the pressure is determined from Bernoulli's equation

$$
p-p_{\infty}+\frac{1}{2} \rho v^{2}=0
$$

In equation (21), the fact that $u$ approaches zero outside the plume has been used. The velocities implied by eq (20) may be determined from eqs (9). Upon substitution into eq (21), the pressure becomes

$$
p-p_{\infty}=-\frac{g Q}{\nu h_{\infty}} \frac{1}{2}\left[\frac{F(\infty)}{\eta}\right]^{2} \frac{1}{\left(\xi^{2}+\frac{1}{4} \eta^{2}\right)} .
$$

Comparing eq (22) with the last of eqs (8), and making use of eq (19), it is easily seen that eq (22) is consistent with the asymptotic plume solution when $\xi \gg \eta$. It is also consistent with the fact, noted in the previous section, that the inertial effects dominate buoyancy contributions to the function $P(\eta)$ for large $\eta$. Finally, the velocities and pressures implied by eqs (20) and (22) satisfy the boundary conditions at infinity given in eq (2). Thus, the velocities given by eqs (9), (13), and (17) constitute a uniformly valid approximation to the velocity for $\xi^{2}+1 / 4 \eta^{2} \gg 1$ (i.e., as $|r|$ approaches infinity in all directions). The corresponding uniform approximation for the pressure is readily seen to be

$$
p-p_{\infty}=\frac{g Q}{\nu h_{\infty}} \frac{P(\eta)}{\left(\xi^{2}+\frac{1}{4} \eta^{2}\right)} .
$$


The flow pattern is illustrated in figures 3 and 4 for $\operatorname{Pr}=0.7$ and 1.0, respectively. These values are chosen because $\operatorname{Pr}=0.7$ is appropriate for most common gases, while $\operatorname{Pr}=1$ is an often used approximation in boundary layer calculations. The heat source is at the origin and the gravity vector points to the left. The bouyant plume occupies the region where the streamlines are approximately parallel to the axis of symmetry. The streamlines well away from the plume are parabolas of constant $\xi$. The transition region is observed to be roughly a parabola of constant $\eta$. The streamlines are all asymptotically parallel, sufficiently far above the heat source.

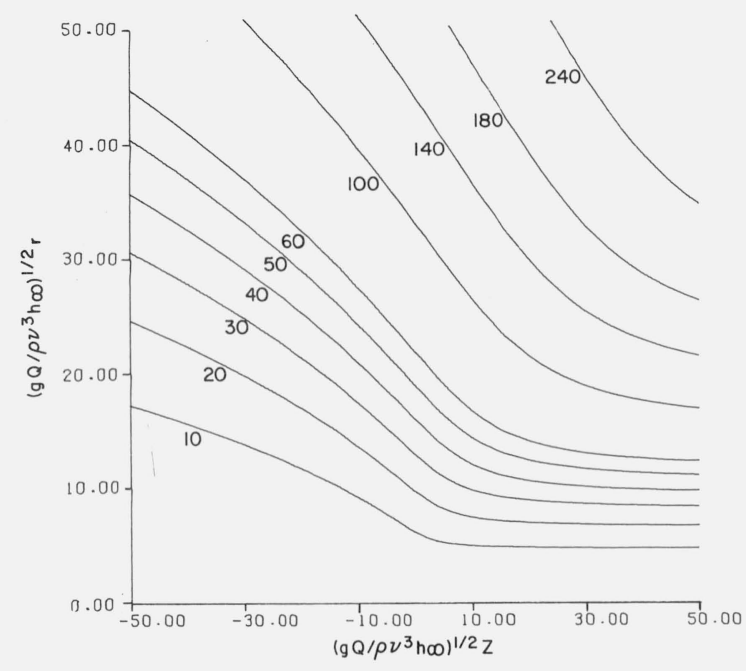

Figure 3. The streamline pattern for several values of the dimensionless stream function $\left(\mathrm{gQ} / \rho \mathrm{h}_{\infty} \nu^{3}\right)^{1 / 2} \Psi / \nu$ and a Prandtl number of 0.7 .

The heat source is at the origin and the gravity vector points to the left.

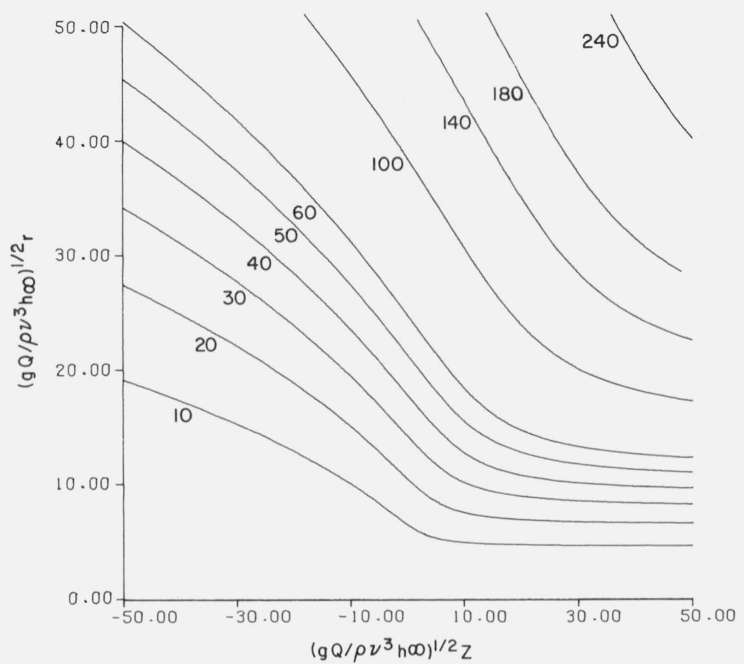

Figure 4. The streamline pattern for several values of the dimensionless stream function $\left(\mathrm{gQ} / \rho \mathrm{h}_{\infty} \nu^{3}\right)^{1 / 2} \Psi / \nu$ and a Prandtl number of 1.0 .

The heat source is at the origin and the gravity vector points to the left.

The corresponding pressure distributions are shown in figures 5 and 6 , respectively. The contours corresponding to $p-p_{\infty}>0$ lie to the right (i.e. above) the heat source. These contours are all closed. They correspond to the positive portion of the $P(\eta)$ curve in figure 2 . This high pressure region is dominated by buoyancy effects. The $p=p_{\infty}$ curve is of course a parabola $\eta=$ constant, the value of $\eta$ being determined by the zero of $P(\eta)$. The remainder of the flow field is characterized by negative values of $p-p_{\infty}$. This region is inertially dominated and as mentioned earlier, is nearly irrotational. The very rapid variation in pressure near the origin is probably spurious, since the assumption that $\xi^{2}+1 / 4 \eta^{2} \gg 1$ is certainly violated there.

The overall flow pattern that emerges is thus determined by the relative magnitudes of the buoyancy and pressure forces. Inside the plume, the bouyancy dominates in the vertical momentum balance, providing the force necessary to overcome the viscous drag and produce the upward motion. The lateral momentum balance is more complex, since the pressure acts first to draw the fluid into the plume, and then to retard and turn it toward the vertical. Outside the plume, the buoyancy rapidly becomes ineffective, since the lateral diffusion of heat is not sufficient to overcome its upward convection to the extent necessary to compete with the pressure forces. The pressure then acts to draw enough fluid into the plume to match the mass flux required at each station to carry the constant energy flux released by the source. 


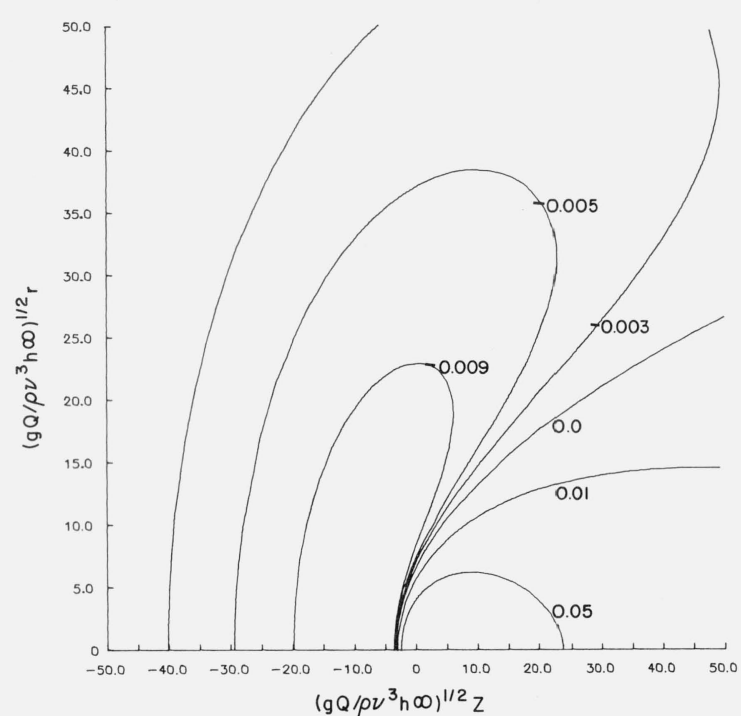

Figure 5. Contours of constant dimensionless overpressure $\mathrm{h}_{\infty}\left(\mathrm{p}-\mathrm{p}_{\infty}\right) / \mathrm{gQ}$ for a Prandtl number of 0.7 .

The heat source is at the origin and the gravity vector points to the left.

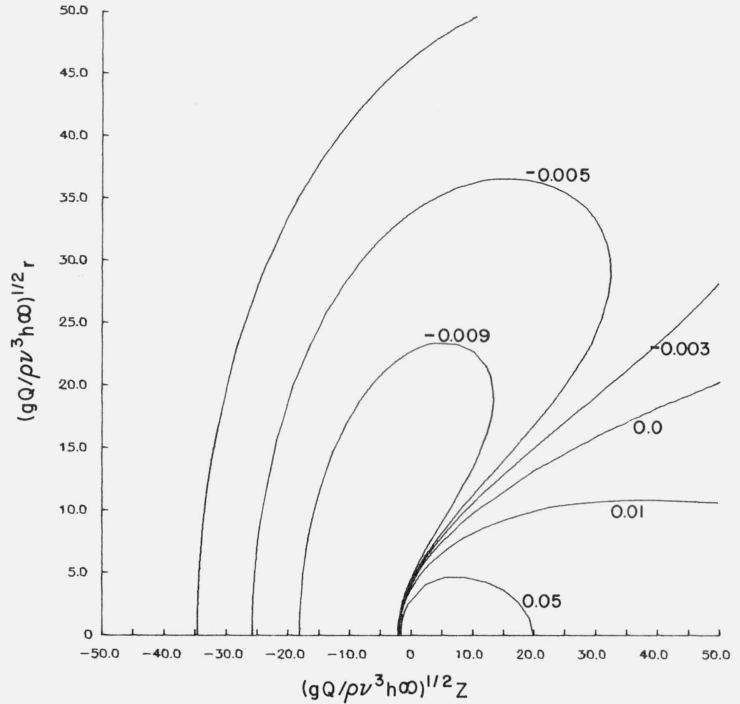

Figure 6. Contours of constant dimensionless overpressure $\mathrm{h}_{\infty}\left(\mathrm{p}-\mathrm{p}_{\infty}\right) / \mathrm{gQ}$ for a Prandtl number of 1.0 .

The heat source is at the origin and the gravity vector points to the left.

The enthalpy outside the plume is not high enough to affect the motion to any significant extent. However, its determination is necessary to verify that the enthalpy distribution in the plume can be joined smoothly to a solution that vanishes as $\xi^{2}+1 / 4 \eta^{2} \rightarrow \infty$ for all $\xi / \eta$. This solution cannot be obtained by the methods used to obtain $\Psi$ and $p-p_{\infty}$. It is therefore necessary to discuss the sense in which these solutions satisfy the equations of motion, so that comparable approximations can be made for the enthalpy. To this end, it should be noted that the plume region corresponds to the region $\phi \rightarrow 0$, where $\phi$ is the polar angle measured from the positive $z$ axis (see fig. 1). This may be seen by noting the transformations

$$
\begin{aligned}
\xi^{2}+\frac{1}{4} \eta^{2} & =\left(\frac{g Q}{\rho h_{\infty} \nu^{3}}\right)^{1 / 2}\left(r^{2}+z^{2}\right)^{1 / 2} \\
\frac{2 \xi}{\eta} & =\cot \left(\frac{\phi}{2}\right) .
\end{aligned}
$$

Thus, the irrotational flow occupies the region $\phi>0$ for distances $\left(r^{2}+z^{2}\right)^{1 / 2}$ sufficiently far from the origin. The actual distance $\left(r^{2}+z^{2}\right)^{1 / 2}$ required for $\xi+1 / 4 \eta^{2} \gg 1$ is dependent upon the parameter $\left(g Q / p h_{\infty} \nu^{3}\right)^{1 / 2}$. The irrotational velocity and pressure were required to match the asymptotic forms of the plume solutions as $\Phi$ and hence $\frac{\eta}{2 \xi}$ vanish. They also were required to vanish at infinity. The same conditions will now be applied to the determination of the enthalpy.

\section{The Uniformly Valid Temperature Distribution}

The solution is based on the fact that outside the plume the stream function $\Psi$ may be approximated by eq (20). The energy equation, eq (7), then becomes:

$$
\begin{gathered}
2 \xi \operatorname{Pr} F(\infty) \frac{\partial H}{\partial \eta}+\frac{1}{2} \frac{\partial}{\partial \xi}\left(\xi \eta \frac{\partial H}{\partial \xi}\right)+2 \frac{\partial}{\partial \eta}\left(\xi \eta \frac{\partial H}{\partial \eta}\right)=0 \\
\theta=\frac{Q}{\rho \nu}\left(\frac{g Q}{\rho \nu^{3} h_{\infty}}\right)^{1 / 2} H(\xi, \eta)
\end{gathered}
$$


$H$ must vanish as $\xi^{2}+1 / 4 \eta^{2} \rightarrow \infty$. As $\phi \rightarrow 0$, the solution must match that given by the second of eqs (19), the asymptotic form of the solution in the plume. These boundary conditions may be written explicitly as:

$$
\begin{aligned}
& H=0 ; \xi^{2}+\frac{1}{4} \eta^{2} \rightarrow \infty \\
& H=\frac{G(\infty)}{\xi^{2}}(\eta)^{-P \gamma F(\infty)} ; \quad \frac{\eta}{\xi} \rightarrow 0 .
\end{aligned}
$$

Once the solution satisfying eqs (23) and (24) is obtained, it will be combined with the solution obtained in section 3 in a straight-forward way to produce the desired result valid for all $\xi^{2}+1 / 4 \eta^{2} \gg 1$.

The solution to eq (23) may be obtained by Hankel Transform techniques. The Hankel Transform $\bar{H}(s, \eta)$ of $H$ is defined by [13]

$$
\begin{aligned}
& \bar{H}(s, \eta)=\int_{0}^{\infty} d \xi \xi J_{0}(\xi s) H(\xi, \eta) \\
& H(\xi, \eta)=\int_{0}^{\infty} d s s J_{0}(\xi s) \bar{H}(s, \eta) .
\end{aligned}
$$

Multiplying eq (23) by the Bessel Function $J_{0}(\xi \mathrm{s})$ and integrating over $\xi$ from zero to infinity yields:

$$
\frac{2}{\eta}\left\{\operatorname{Pr} F(\infty) \frac{\partial \bar{H}}{\partial \eta}+\frac{\partial}{\partial \eta}\left(\eta \frac{\partial \bar{H}}{\partial \eta}\right)\right\}-\frac{s^{2}}{2} \bar{H}=0
$$

The solution of eq (26) satisfying the boundary condition at infinity is

$$
\bar{H}=(\eta s)^{-\lambda} K_{\lambda}\left(\frac{\eta s}{2}\right) h(s) .
$$

Here $K_{\lambda}$ is the Modified Bessel Function of order $\lambda[14]$; and $\lambda$ is defined in eq (14). Thus, from eq $(25)$ :

$$
H(\xi, \eta)=\int_{0}^{\infty} d s s J_{0}(\xi s) h(s)(\eta s)^{-\lambda} K_{\lambda}\left(\frac{\eta s}{2}\right)
$$

The function $h(s)$ must be determined from the matching boundary condition given in the second of eqs (24). This boundary condition can be applied to eq (28) in a straight-forward manner if boundary conditions are known as $\eta \rightarrow 0$ for all $\xi$. Thus, letting $\eta \rightarrow 0$ in eq (28) with $\xi$ fixed by using the limiting form of $K_{\lambda}(\eta s / 2)$ for small values of its argument:

$$
H(\xi, \eta)=\left(\frac{1}{2}\right)^{1-2 \lambda} \Gamma(\lambda)(\eta)^{-2 \lambda} \int_{0}^{\infty} d s J_{0}(\xi s)(s)^{1-2 \lambda} h(s)
$$

Here, $\Gamma$ denotes the Gamma Function [14]. The second of eqs (24) is now temporarily replaced by the following boundary condition, which is assumed to hold for all $\xi$ as $\eta \rightarrow 0$ :

$$
H=\frac{G(\infty)}{\xi^{2}+1}(\eta)^{-2 \lambda}
$$

Equating (29) and (30):

$$
\begin{gathered}
(2)^{1-2 \lambda} \frac{G(\infty)}{\Gamma(\lambda)\left(\xi^{2}+1\right)}=\int_{0}^{\infty} d s s J_{0}(\xi s) h^{*}(s) \\
h^{*}(s)=s^{-2 \lambda} h(s)
\end{gathered}
$$


Equation (31) can be readily inverted making use of a special case of the Modified WeberSchafheitlein integral [15].

Thus

$$
\int_{0}^{\infty} K_{0}(a s) J_{0}(\xi s) s d s=\frac{1}{\xi^{2}+a^{2}}
$$

$$
h^{*}=(2)^{1-2 \lambda} \frac{G(\infty)}{\Gamma(\lambda)} K_{0}(s) .
$$

Finally, using the inversion integral (25):

$$
H(\xi, \eta)=\frac{(2)^{1-2 \lambda}}{\Gamma(\lambda)} G(\infty) \int_{0}^{\infty} d s s K_{0}(s)\left(\frac{s}{\eta}\right)^{\lambda} K_{\lambda}\left(\frac{\eta s}{2}\right) J_{0}(s \xi) .
$$

Equation (34) constitutes an acceptable solution to the problem, since the matching condition, eq (24), is contained in the boundary condition actually satisfied. They become identical when $\xi \gg 1$. However, there is a certain arbitrariness in eq (34). There are many functions which are regular for all $\xi$ and are proportional to $(\xi)^{-2}$ for $\xi \gg 1$. Any such expression, when used in place of the factor $\left(\xi^{2}+1\right)^{-1}$ in eq (30), would give a different solution for $h^{*}(s)$. It would be expected, however, that the behavior of $h^{*}(s)$ as $s \rightarrow 0$ would be identical for all such choices, since the $\xi^{-2}$ behavior as $\xi \rightarrow \infty$ must be preserved if the matching condition is to be satisfied. The arbitrariness can be removed by considering the limiting form of eq (34) when $\xi^{2}+1 / 4 \eta^{2}$ is large, but $\xi / \eta$ is arbitrary. This is accomplished by replacing the function $K_{o}(s)$ by its limiting form for small $s$. The result is

$$
H(\xi, \eta)=\frac{-(2)^{1-2 \lambda}}{\Gamma(\lambda)} G(\infty) \int_{0}^{\infty} d s s \log s\left(\frac{s}{\eta}\right)^{\lambda} K_{\lambda}\left(\frac{\eta s}{2}\right) J_{0}(s \xi) .
$$

Now eq (35) is necessarily an exact solution of eq (23) since only $h(\mathrm{~s})$ has changed. It also satisfies the boundary conditions, eqs (24), as will be demonstrated below.

Equation (35) can be rewritten in the form:

$$
\begin{aligned}
H(\xi, \eta) & =\left.\frac{-(2)^{1-2 \lambda}}{\Gamma(\lambda)} G(\infty)(\eta)^{-\lambda} \frac{\partial}{\partial p} J(\xi, \eta, p)\right|_{v=1+\lambda} \\
J & =\int_{0}^{\infty} d s(s)^{p} K_{\lambda}\left(\frac{\eta s}{2}\right) J_{0}(s \xi)
\end{aligned}
$$

Using the general form of the Modified Weber-Schafheitlein integral [15]:

$$
\begin{aligned}
& J=(2)^{2 p}(\eta)^{-(1+p)} \Gamma(a) \Gamma(b)_{2} F_{1}\left(a, b, 1,-\frac{4 \xi^{2}}{\eta^{2}}\right) \\
& a=\frac{1}{2}(p+\lambda+1) \\
& b=\frac{1}{2}(p-\lambda+1) .
\end{aligned}
$$

The quantity ${ }_{2} \mathrm{~F}_{1}$ is the Hypergeometric function [14]. Carrying out the differentiation, eq (36) becomes:

$$
\begin{aligned}
H=\frac{-8 G(\infty) \Gamma(\lambda+1)}{\Gamma(\lambda)}\left[\left\{\log \left(\frac{4}{\eta}\right)+\frac{1}{2}[\Psi(1)+\Psi(1+\lambda)]\right\}\left(\eta^{2}+4 \xi^{2}\right)^{-(1+\lambda)}\right. & \\
& \left.+\left.(\eta)^{-2(1+\lambda)} \frac{\partial}{\partial p}\left\{{ }_{2} F_{1}\left(a, b, 1,-\frac{4 \xi^{2}}{\eta^{2}}\right)\right\}\right|_{p=1+\lambda}\right]
\end{aligned}
$$

The quantity $\Psi(z)$ in eq (38) is the Digamma Function [14]; and the following result has been used:

$$
{ }_{2} F_{1}(a, b, b ; z)=(1-z)^{-a} \text {. }
$$


In order to carry out the differentiation of ${ }_{2} F_{1}\left(a, b, 1,-4 \xi^{2} / \eta^{2}\right)$, it is necessary to have an explicit representation of this function. The Barnes integral [14] is convenient, but can only be used to describe ${ }_{2} F_{1}(a, b, c, z)$ for $c>b>0$. Accordingly, the Gauss relations are used in the form:

$$
{ }_{2} F_{1}(a, b, 1, z)=\frac{1}{1-z}\left\{[a-1-(1-b) z]_{2} F_{1}(a, b, 2, z)+(2-a){ }_{2} F_{1}(a-1, b, 2, z)\right\} .
$$

Thus, since $b$ is in the neighborhood of 1 in the domain of interest, the Barnes integral can be used on the right-hand side of eq (39) to obtain:

$$
\begin{gathered}
{ }_{2} F_{1}(a, b, 1, z)=(1-z)^{-1}\{\Gamma(b) \Gamma(2-b)\}^{-1} . \\
\left\{[(a-1)-(1-b) z] \int_{0}^{1} d x\left(\frac{x}{1-x}\right)^{b-1}(1-x z)^{-a}+(2-a) \int_{0}^{1} d x\left(\frac{x}{1-x}\right)^{b-1}(1-x z)^{-(a-1)}\right\} .
\end{gathered}
$$

Thus:

$$
\begin{aligned}
&\left.\frac{\partial}{\partial p}{ }_{2} F_{1}(a, b, 1, z)\right|_{p=1+\lambda}=[2(1-z)]^{-1} \\
& \times\left\{(\lambda z)^{-1}(1+z)\left[(1-z)^{-\lambda}-1\right]-[(\lambda-1) z]^{-1}\left[(1-z)^{-(\lambda-1)}-1\right]\right. \\
&-(\lambda z)^{-1}\left\{\left[(1-z)^{-\lambda}-1\right]+\lambda(1-z)^{-\lambda} \log (1-z)\right\} \\
&+ {[(\lambda-1) z]^{-1}\left\{\left[(1-z)^{-(\lambda-1)}-1\right]+(\lambda-1)(1-z)^{-(\lambda-1)} \log (1-z)\right\} } \\
&\left.+\lambda \int_{0}^{1} d x \log \left(\frac{x}{1-x}\right)(1-x z)^{-(\lambda+1)}+(1-\lambda) \int_{0}^{1} d x \log \left(\frac{x}{1-x}\right)(1-z x)^{-\lambda}\right\} .
\end{aligned}
$$

Inserting eq (41) into eq (38) with $z=-4 \xi^{2} / \eta^{2}$; the following expression is obtained:

$$
\begin{aligned}
& H=\frac{G(\infty)(\eta)^{-2 \lambda}}{\left(\xi^{2}+\frac{1}{4} \eta^{2}\right)}\left\{2 \lambda \log \left(\frac{\eta}{4}\right)\left(1+t^{2}\right)^{-\lambda}+f(t)\right\} \\
& f(t)=-\lambda[\Psi(1)+\Psi(\lambda+1)]\left(1+t^{2}\right)^{-\lambda}+\frac{\left(t^{2}-1\right)}{t^{2}}\left[1-\left(1+t^{2}\right)^{-\lambda}\right] \\
&+\lambda\left[(\lambda-1) t^{2}\right]^{-1}\left[1-\left(1+t^{2}\right)^{-(\lambda-1)}\right] \\
&+\frac{2}{t^{2}}\left\{1-\left(1+t^{2}\right)^{-\lambda}-\lambda\left(1+t^{2}\right)^{-\lambda} \log \left(1+t^{2}\right)\right\} \\
&+2 \lambda\left[(\lambda-1) t^{2}\right]^{-1}\left\{\left(1+t^{2}\right)^{-(\lambda-1)}-1+(\lambda-1)\left(1+t^{2}\right)^{-(\lambda-1)} \log \left(1+t^{2}\right)\right\} \\
&-\frac{\lambda}{t^{2}}\left\{\left(1+t^{2}\right)^{-\lambda} \int_{0}^{t^{2}} \frac{d x}{x}\left[(1+x)^{\lambda}-1\right]-\int_{0}^{t^{2}} \frac{d x}{x}\left[1-(1+x)^{-\lambda}\right]\right\} \\
&+\frac{\lambda}{t^{2}}\left\{\left(1+t^{2}\right)^{-(\lambda-1)} \int_{0}^{t^{2}} \frac{d x}{x}\left[(1+x)^{\lambda-1}-1\right]-\int_{0}^{t^{2}} \frac{d x}{x}\left[1-(1+x)^{-(\lambda-1)}\right]\right\} \\
& t=\frac{2 \xi}{\eta}=\cot \left(\frac{\phi}{2}\right) .
\end{aligned}
$$

The demonstration that the boundary conditions, eqs (24) are satisfied can be made using eq (42). Since $t$ depends only on the polar angle $\phi, H$ obviously vanishes as $\xi^{2}+1 / 4 \eta^{2} \rightarrow \infty$ for any non-zero value of $\phi$ (finite $t$ ). Furthermore, $f(t)$ has the limiting behavior

$$
\begin{aligned}
& f(t)=-\lambda[\Psi(1)+\Psi(\lambda+1)]+O\left(t^{2}\right) ; \quad t \rightarrow 0 \\
& f(t)=1+O\left(t^{-2}\right) ; \quad t \rightarrow \infty .
\end{aligned}
$$


As the plume region is approached $(\phi \rightarrow 0 ; t \rightarrow \infty)$, the matching boundary condition is recovered.

$$
H=\frac{G(\infty)(\eta)^{-2 \lambda}}{\xi^{2}}\left\{1+O\left(\frac{\eta^{2}}{\xi^{2}}\right)\right\}
$$

Finally, a uniform approximation to $H(\xi, \eta)$ valid for all $\phi$ may be constructed. Note that the quantity $G(\infty)(\eta)^{-2 \lambda}$ appearing in eq (42) is the asymptotic form of the plume enthalpy function $G(\eta)$. Now consider the form

$$
H(\xi, \eta)=\frac{G(\eta)}{\left(\xi^{2}+\frac{1}{4} \eta^{2}\right)}\left\{\lambda \log \left[\left(\frac{\eta}{4}\right)^{2}+1\right]\left(1+t^{2}\right)^{-\lambda}+f(t)\right\} .
$$

Applying the limit $\xi \rightarrow \infty$ with $\eta$ fixed to eq (43), the form for $h-h_{\infty}$ given by eq (8) is recovered. The limit $\xi+\frac{1}{4} \eta^{2} \rightarrow \infty$ with $\phi$ fixed yields eq (42). The replacement of $2 \log (\eta / 4)$ by $\log \left[(\eta / 4)^{2}+1\right]$ is motivated by the desire to have the uniform expansion be an analytic function of $\eta^{2}$.

The function $f(t)$ is plotted as a function of polar angle $\phi(\mathrm{eq}(42))$ in figure 7 . The function is nearly constant over the region occupied by the plume, but varies rapidly elsewhere. Figures 8 and 9 illustrate the enthalpy distribution, showing several level curves of the function $H(\xi, \eta)$ given by eq (43). Again, the detailed structure near the origin cannot be taken seriously, due to the smallness of $\xi^{2}+1 / 4 \eta^{2}$. The figure does illustrate, however, the rapidity with which the excess enthalpy decreases away from the plume region near the positive $z$ axis.

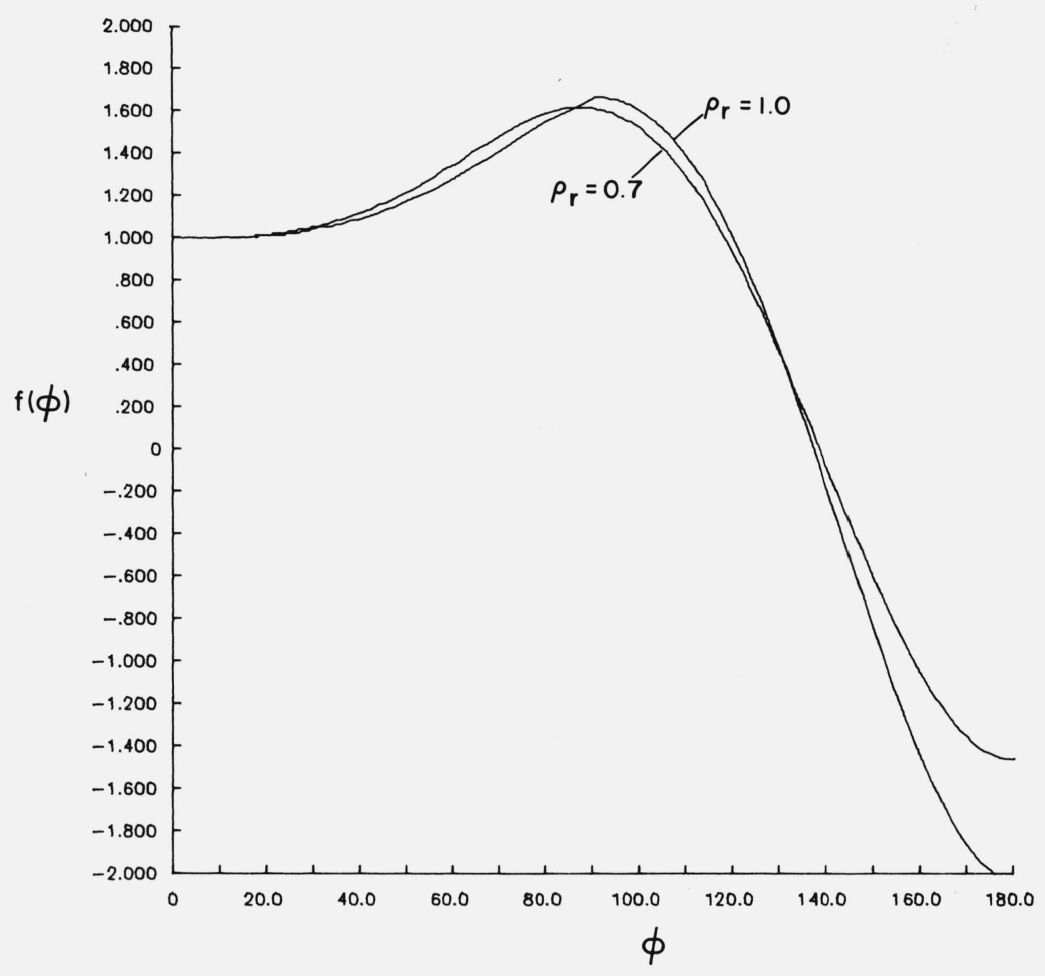

FIGURE 7. The function $\mathrm{f}(\phi)$ appearing in eq (43) plotted as a function of polar angle measured in degrees. $\phi=0$ corresponds to the positive axis (the plume centerline). 


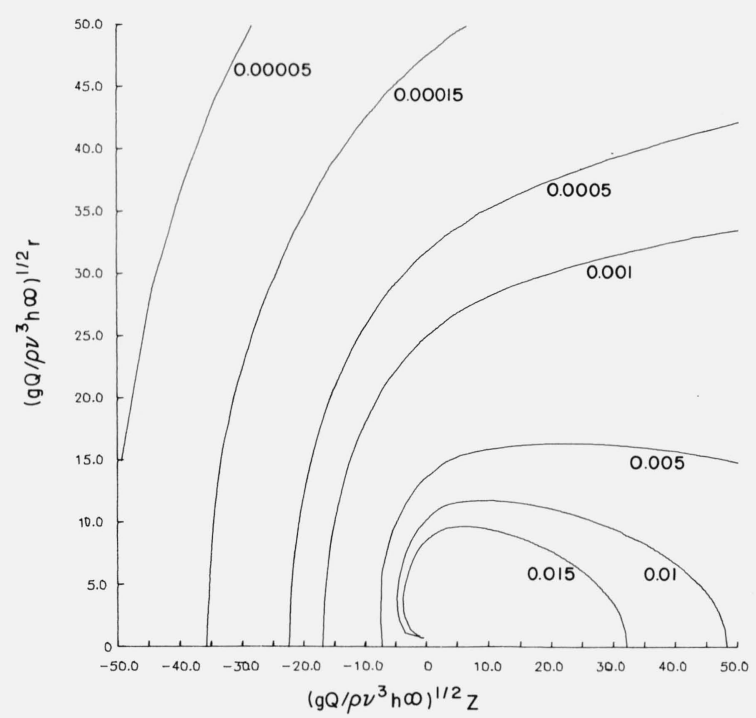

Figure 8. Contours of constant dimensionless enthalpy rise $\rho \nu\left(\rho \nu^{3} \mathrm{~h}_{\infty} / \mathrm{gQ}\right)^{1 / 2}\left(\mathrm{~h}-\mathrm{h}_{\infty}\right) / \mathrm{Q}$ for a Prandtl number of $0 . \%$.

The heat source is at the origin and the gravity vector points to the left.

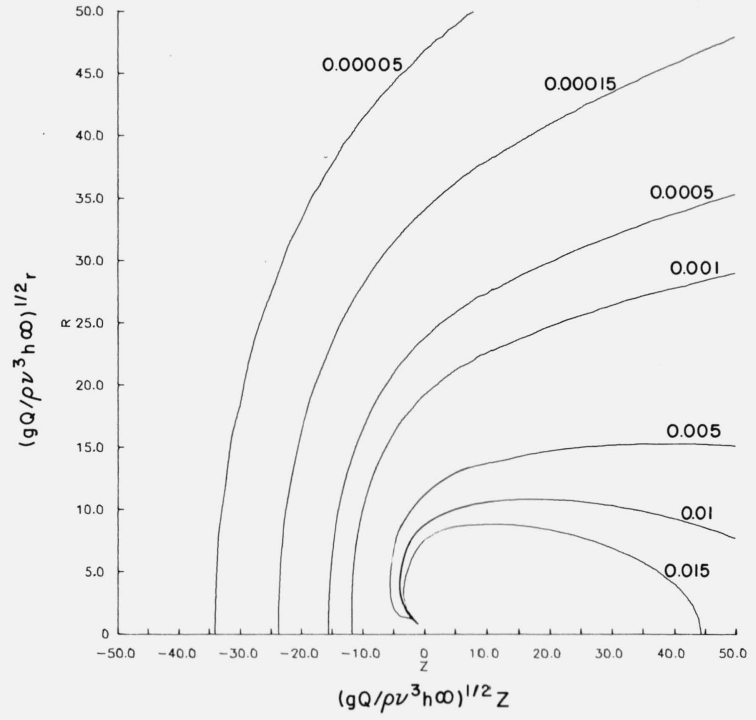

Figure 9. Contours of constant dimensionless enthalp y rise $\rho \nu\left(\rho \nu^{3} \mathrm{~h}_{\infty} / \mathrm{gQ}^{1 / 2}\left(\mathrm{~h}-\mathrm{h}_{\infty}\right) / \mathrm{Q}\right.$ for a Prandtl number of 1.0 .

The heat source is at the origin and the gravity vector points to the left

\section{Discussion}

The present results shed some light on the use of the Oseen approximation in buoyancy induced flow problems. In the present context, the "Oseen approximation" is taken to mean the replacement of the convection velocity by a substitute quantity whose choice simplifies the calculation of the flow quantities of interest [16]. As noted in the introduction, Fendell [11] and Wesseling [12] use this technique to study convection induced by a localized heat source. Wesseling assumes the heat source to be at rest in a uniform stream produced by some external agent, whereas Fendell's motion is induced entirely by the heat source.

First consider Fendell's analysis. Here, the convection velocity is assumed to be the centerline plume velocity, with the result that the velocity and temperature perturbations decay exponentially with distance away from the plume. The actual convection velocity outside the plume is given by the second of equations (9) with $F(\eta)=F(\infty)$. This velocity itself decays slowly in all directions, and is much less effective at inhibiting the diffusion of heat away from the plume than a constant velocity parallel to the plume would be.

The analysis contained in the previous section may be thought of as an Oseen linearization with the irrotational flow as the convection velocity. The irrotational flow is dominant in this region, as discussed in section 4 . However, there remains a residual vorticity containing motion outside the plume, analogous to the enthalpy distribution calculated in section 5 . This motion could in principal be calculated by applying an Oseen linearization to the vorticity equation with the convection velocity described above. In the author's view, such a calculation would miss the main point; that the dominant motion outside the plume is irrotational. This irrotational motion is not known a priori and must be determined from a knowledge of the plume solution, including the pressure distribution. Once it is known, the homogeneous solution to the vorticity equation, corresponding to $\omega=0$, is known. The solution $\omega=0$ thus implies the non-trivial flow discussed in section 4. It is the existence of the irrotational flow which distinguishes the solution $\omega=0$ of the vorticity equation from the trivial solution $h-h_{\infty}=0$ of the energy equation. This is why the small quantity $h-h_{\infty}$ must be calculated outside the plume, while the corresponding vorticity distribution is unnecessary unless desired for its own sake. 
The preexisting uniform flow in the absence of any heat source is used as the convection velocity in Wesseling's analysis. The consistency of his result then requires the buoyancy induced velocities to be small compared with the uniform flow everywhere away from the source. Two cases are considered; a point source and a line source. Wesseling finds that while the point source has an acceptable solution, the line source does not. He remarks "it remains to be seen whether the Stokes-type paradox exhibited above is caused by the Oseen linearization or whether it is a property of the full Boussinesq equations." Although it is not possible to settle this question here, the following facts are suggestive. The present analysis together with previous work demonstrate that the thermally induced velocity in a medium at rest is bounded everywhere away from a point source. On the other hand, the velocity of a plume generated by a line source in a medium at rest increases with the $1 / 5$ power of the distance above the source. This must induce a velocity outside the plume which is ultimately larger than any uniform velocity. Thus, the thermally induced velocity probably dominates the motion outside the plume in a moving medium in two dimensions. This velocity cannot be assumed a priori as was done by Wesseling, but must be determined. If detailed analysis bears out the above hypothesis, then it is the Oseen linearization which must be reconsidered in two dimensions rather than the Boussinesq equations.

The author is indebted to Margaret Harkleroad for the numerical computations and the figures appearing in this work.

\section{References}

[1] Schuh, H., Boundary layers of temperature, Boundary Layers, W. Tollmien, Ed. British Ministry of Supply, German Document Center, Ref. 3220 T, Sec. B6 (1948).

[2] Yih, C. S., Free convection due to a point source of heat. Proceedings of the first U.S. National Congress of Applied Mechanics, Illinois Inst. Technology, 941 (1952).

[3] Fujii, T., Theory of the steady laminar natural convection above a horizontal line heat source and a point heat source, Int. J. Heat Mass Transfer 6, 597 (1963).

[4] Brand, R. S., and Lahey, F. J., The heated laminar vertical jet, J. Fluid Mech. 29, 305 (1967).

[5] Gebhart, B., Natural convection flows and stability, Advances in Heat Transfer, T. F. Irvine and J. P. Hartnett, Eds., 9 (Academic Press, New York, 1973).

[6] Schlichting, H., Laminare Strahlausbreitung, ZAMM 13, 260 (1933).

[7] Bickley, W., The plane jet, Phil. Mag. Ser. 7, 23, 727 (1939).

[8] Schlichting, H., Boundary Layer Theory (McGraw-Hill, New York, 1955).

[9] Landau, L. D., and Lifshitz, E. M., Fluid Mechanics (Addison-Wesley, Reading, 1955), p. 86.

[10] Goldstein, S., Lectures on Fluid Mechanics, (Interscience, New York, 1960), p. 131.

[11] Fendell, F. E., Laminar natural convection about an isothermally heated sphere of small Grashof number, J. Fluid Mech. 34, 163 (1968).

[12] Wesseling, P., An asymptotic solution for slightly buoyant laminar plumes, J. Fluid Mech. 70, 81 (1975).

[13] Sneddon, I. N., Fourier Transforms (McGraw-Hill, New York, 1951).

[14] Abramowitz, M. and Stegun, I. A., Handbook of Mathematical Functions (U.S. Government Printing Office, 1966).

[15] Watson, G. N., Theory of Bessel Functions (Cambridge University Press, Cambridge, 1944 ), p. 410.

[16] Carrier; G. F., Analytic approximation techniques in applied mathematics, J. Soc. Indust. App. Math. 13, 68 (1965).

(Paper 81B 1 \& 2-461) 